\title{
Interacting thermofield doubles and critical behavior in random regular graphs
}

\author{
O. Valba $\oplus^{1,2}$ and A. Gorsky ${ }^{3,4}$ \\ ${ }^{1}$ Department of Applied Mathematics, National Research University Higher School of Economics, \\ Moscow 101000, Russia \\ ${ }^{2}$ Federal Research Center of Chemical Physics RAS, Moscow 119991, Russia \\ ${ }^{3}$ Institute of Information Transmission Problems RAS, Moscow 127051, Russia \\ ${ }^{4}$ Moscow Institute of Physics and Technology, Dolgoprudny 141700, Russia
}

(Received 31 March 2021; accepted 12 April 2021; published 11 May 2021)

\begin{abstract}
We discuss numerically the nonperturbative effects in exponential random graphs which are analogue of eigenvalue instantons in matrix models. The phase structure of exponential random graphs with chemical potential for $C_{4} \mu_{4}$ and degree preserving constraint is clarified. The first order phase transition at critical value of chemical potential for $C_{4} \mu_{4}^{\mathrm{RRG}}$ into bipartite phase with a formation of fixed number of bipartite clusters is found for ensemble of random regular graphs (RRG). We consider the similar phase transition in mean field version of combinatorial quantum gravity based of the Ollivier graph curvature for RRG supplemented with hard-core constraint and show that a order of a phase transition at $\mu_{4}^{\mathrm{CRRG}}$ and the structure of emerging phase depend on a vertex degree $d$ in RRG. For $d=3$ the bipartite closed ribbon emerges at $\mu_{4}>\mu_{4}^{\mathrm{CRRG}}$ while for $d>3$ the ensemble of isolated or weakly interacting hypercubes supplemented with the bipartite closed ribbon gets emerged at the first order phase transition with a clearcut hysteresis. If the additional connectedness condition is imposed the phase at $\mu_{4}>\mu_{4}^{\mathrm{CRRG}}$ gets identified as the closed chain of weakly coupled hypercubes. Since the ground state of isolated hypercube is the thermofield double we suggest that the dual holographic picture involves multiboundary wormholes. Treating RRG as a model of a Hilbert space for a interacting many-body system we discuss the patterns of the Hilbert space fragmentation at the phase transition. We also briefly comment on a possible relation of the found phase transition to the problem of holographic interpretation of a partial deconfinement transition in the gauge theories.
\end{abstract}

DOI: $10.1103 /$ PhysRevD.103.106013

\section{INTRODUCTION}

Matrix models play a prominent role in a description of chaotic systems with large number degrees of freedom. They govern, for instance, a spectrum of a chaotic system, the space-time dynamics of some microscopic degrees of freedom, discretization of the Riemann surfaces or their moduli spaces [1]. The different ensembles of random matrices correspond to systems with particular symmetry patterns. Spectral density, spectral correlators, and level spacing distribution are among the most popular characteristics within the matrix model approach.

Large $N$ matrix models enjoy the nonperturbative phenomena: eigenvalue instantons that are suppressed as

Published by the American Physical Society under the terms of the Creative Commons Attribution 4.0 International license. Further distribution of this work must maintain attribution to the author(s) and the published article's title, journal citation, and DOI. Funded by SCOAP ${ }^{3}$. $\exp (-N)$ [2,3]. The partition function for some matrix ensemble reads as

$$
Z\left(t_{2}, t_{3} \ldots t_{n}\right)=\int d M \exp \left(-\sum_{k}^{n} t_{k} \operatorname{Tr} M^{k}\right)
$$

where the effective potential for the eigenvalues in the integration measure generically has several extrema. In the eigenvalue matrix model the ground state is determined by the distribution of eigenvalues of $M$ among extrema of effective potential which depends on the variables $t_{k}$. If the term $t_{2}$ dominates the eigenvalues typically collected at one extremum, then the eigenvalue instanton corresponds to the traveling of the single eigenvalue from this extremum to another one. The initial symmetry like $S U(n)$ is broken down to some subgroup if multiple eigenvalue instantons are taken into account. Such symmetry breaking effects are important at the phase transitions at some critical values of $t_{k}$ in the matrix models with different matrix measures. From the physical viewpoint eigenvalue instantons 
correspond to baby-universe in 2D gravity, domain walls in supersymmetric Yang-Mills (SYM) theory or ZZ branes in Liouville theory. The review of the different manifestations of eigenvalue instantons can be found in $[4,5]$.

Exponential random graphs are statistical models with the partition function

$$
Z\left(\mu_{2}, \mu_{3} \ldots \mu_{n}\right)=\sum_{\text {graphs }} \exp \left(-\sum_{k}^{n} \mu_{k} \operatorname{Tr} A^{k}\right),
$$

where $A$ is a graph adjacency matrix and the summation runs over some ensemble, say, over Erdős-Rényi (ER) ensemble, which involves the potential $\operatorname{Tr} A^{2}$ and is the network analogue of Gaussian matrix model. The sum over ensembles of graphs substitutes the integration over matrix ensembles in matrix models. The terms $\mu_{k} \operatorname{Tr} A^{k}$ in the measure are similar to $t_{k} \operatorname{Tr} M^{k}$ terms in the measure of matrix models and $\mu_{k}$ provide chemical potentials for number of higher $k$ cycles in the particular realization. One can consider the dependence of partition function on the chemical potentials and look for the phase transitions caused by condensation of some motifs that are marked by the order parameters $\left\langle\operatorname{Tr} A^{k}\right\rangle \neq 0$ [6-17]. The condensation of links, nodes, and triangles has been considered and these phase transitions are analogs of the familiar criticalities in the conventional matrix models. It was shown that the phase transitions are sensitive to the finite-size effects $[10,17,18]$ and the details of the emerging phases are $N$ dependent in several cases.

The additional local constraint imposing strict degree conservation yields the additional flavor for the condensation phenomena [11,16,17] (see also [19,20] for some earlier observations). It is in this case the eigenvalue instantons enter the game. It turns out that degree conservation constraint amounts to the first order phase transition with formation of the multiple weakly interacting clusters which has been demonstrated for constrained ER and random regular graphs (RRG) with Hamiltonian involving $\mu_{3} \neq 0$ [11]. The local degree conservation constraint in the graph ensemble has a matrix model counterpart as well, it means that nonsinglet sector of a matrix model now matters.

The phase transition can be effectively analyzed in terms of network spectrum. In the ER model with local constraint and RRG the formation of network cluster corresponds exactly to the single eigenvalue instanton. Eigenvalue instantons upon the averaging over ensemble form the new nonperturbative soft band in the spectrum of the graph Laplacian. The similar effects take place in a spectral density of a matrix model upon the account of multiple instantons. Soft "cluster" band is separated with the gap from the perturbative part of the spectrum. The number of instantons equals to the number of clusters. Moreover, the spectrum of the perturbative band gets deformed at the phase transition and acquires the triangular shape [11] typical for scale-free graphs due to the intercluster interactions.

Following parallels with the matrix models the exponential random graphs have been also used as the model for the discrete approximation to quantum gravity in [21]. This approach based on the Ollivier graph curvature was nicknamed as combinatorial quantum gravity. The graph Ollivier curvature $[22,23]$ gets reduced to the Ricci curvature in the continuum limit for the large family of graph ensembles [24] including RRG therefore the EinsteinHilbert action can be recovered. The dimension of emerging Einstein-Hilbert action $D$ is defined by the degree $d$ in RRG and $\mu_{4}$ upon proper rescaling plays a role of the gravitational coupling. It was claimed in $[21,25]$ that such model undergoes the second order phase transition into the dense phase at some $\mu_{4}^{c}$ without a cluster formation. The $d=3$ example has been investigated in [26] and the emerging $S^{1}$ geometry has been identified.

Another popular application of RRG concerns its role as a toy model for the Hilbert space of interacting many-body system. The RRG mimics the realization of the Hilbert space as the Fock space. It was suggested in [27] that oneparticle localization in the Fock space is related to the localization in the interacting many-body system. It was argued in $[28,29]$ that indeed localization $(\mathrm{MBL})$ in the interacting many-body system can be mapped to the problem of one-particle localization in the Fock space with flat diagonal disorder. In [30] we have treated the oneparticle problem in the Fock space without the diagonal disorder but with the structural disorder induced by chemical potential for three cycles. It was found that above the phase transition the states in the nonperturbative band get localized while all states in the perturbative band are delocalized. More recently RRG with diagonal disorder has been analyzed in [31-36], moreover the arguments have been presented that RRG plays the role of tricritical point in the space of deformations [36].

In this paper we investigate numerically the effects of eigenvalue instantons in the graph ensembles with degree conservation. Three graph ensembles are considered:

(i) RRG ensemble, (ii) RRG + hard-core constraint, and (iiiRRG + hard-core constraint + connectedness constraint. First we consider numerically RRG perturbed by chemical potential $\mu_{4}$ for $C_{4}$. It is found that at $\mu_{4}^{\mathrm{RRG}}$ the network gets clusterized however in contrast to the case of three cycles all clusters are bipartite. Then we elaborate the model with the additional hard-core constraint for a possible touching of neighbor $C_{4}$ in RRG suggested in [21], which we denote as CRRG. The nature of the phase transition in CRRG and the dependence on initial configurations is investigated. We find the first order phase transition in CRRG and check the clearcut hysteresis that confirms the order of phase transition for $d>3$. A bit surprisingly it turned out that almost all emerging bipartite clusters are isolated or weakly interacting hypercubes, while the second ingredient of the emerging 
clusterized phase for all numerically available degrees is the composite bipartite closed ribbon. The number of nodes involved into the closed ribbon depends on $\mu_{4}$. Our finding fits with the claim in $[21,25,26]$ about the order of the phase transition for $d>3$ in the mean field approximation.

Finally we introduce the second constraint for CRRG assuming the connectedness of the graph and obtain the closed chain of the weakly connected hypercubes at $\mu_{4}>\mu_{4}^{\mathrm{CRRG}}$. We reproduce the result of [26] for $d=3$ that at $\mu_{4}>\mu_{4}^{\mathrm{CRRG}}$ the single composite bipartite closed ribbon emerges. However for $d>3$ we clearly demonstrate that the clustering into hypercubes occurs in the mean field approximation of combinatorial quantum gravity.

The hypercube phase provides the interesting insight on the possible dual holographic picture. Indeed it was noted in [37] that the hypercube adjacency matrix exactly coincides with the interaction term in the Maldacena-Qi model of two coupled Sachdev-Ye-Kitaev (SYK) models [38]. Hence for a single hypercube we deal with the strong coupling limit of the Maldacena-Qi model, which has thermofield double state (TFD) as the ground state [37,39]. On the other hand the TFD is holographycally dual to the eternal black hole [40]. The eternal black hole was considered as the simple example of the "geometry from entanglement" approach [41] when the dual classical geometry emerges from the entanglement of states of the boundary theory. Here we have the highly entangled Majorana dimers which correspond to the ground state of individual hypercube.

At strong coupling phase we obtain the ensemble of hypercubes which can be completely isolated or weakly interacting. In the holographic picture it fits with the recent discussion $[42,43]$ of the holography for the ensemble of theories at multiple bulk boundaries. If the components do not interact the corresponding holographic geometry is assumed to be multiboundary nontraversable wormhole [44], which becomes traversable if the interaction between the components is added. The multiple Majorana dimers were also used recently for the toy model for holographic quantum error-correcting codes [45].

We shall also make a brief discussion concerning the possible application of our findings for two more problems. In the dimensionally reduced SYM theory down to matrix quantum mechanics, the clusterization of the matrices into the block diagonal form corresponds to the partial breaking of the $S U(N)$ gauge symmetry down to $S U(M)^{k}$ symmetry [46,47]. First, we shall compare our findings in RRG with this interpretation. On the other hand this partial breaking of the gauge symmetry presumably corresponds [47] to the transforming of a graviton gas into small black holes. We shall also speculate on the relevance of the eigenvalue instantons for the partial deconfinement phenomena. Second, we make some comments on the possible interpretation of the emerging new objects: hypercubes and composite bipartite ribbons in the context of Fock space picture for Hilbert space of interacting many-body system.
The phase transition provides the different patterns for the fragmentation of the Hilbert space.

The paper is organized as follows. In Sec. II, we present the results of numerical study of RRG perturbed by chemical potential for $C_{4}$ and supplemented with the different constraints. In Sec. III, the arguments concerning the holographic interpretation of the strong coupling phase are presented. Section IV is devoted to the aspects of Fock space realization of the Hilbert space of many-body system in terms of RRG. The phase transition is considered as a fragmentation of the Hilbert space. The results and the open questions are collected in Sec. V.

\section{PHASE TRANSITIONS IN PERTURBED RANDOM REGULAR GRAPHS}

\section{A. The model description}

Similar to discussion in [11] suppose that we start with some network and rewire links under the condition that at each step of rewiring we try to maximize the number of $C_{4}$ $N_{4}$. Which is the equilibrium structure of the entire network? In mathematical terms this question reads as follows. We assign the chemical potential $\mu_{4}$ to each $C_{4}$ and consider the partition function

$$
Z\left(\mu_{4}\right)=\sum_{\{\text {states }\}} e^{-\mu_{4} N_{4}},
$$

where prime in (3) means that the summation runs over all possible configurations of links ("states"), under the condition of fixed degrees $\left\{v_{1}, \ldots, v_{N}\right\}$ in all network vertices.

To simulate the rewiring process, one applies the standard Metropolis algorithm with the following rules: (i) if under the reconnection the number of $C_{4}$ is increasing, a move (rewiring) is accepted, (ii) if the number of $C_{4}$ is decreasing by $\Delta N_{4}$, or remains unchanged, a move is accepted with the probability $e^{-\mu \Delta N_{4}}$. The Metropolis algorithm runs repeatedly for large set of randomly chosen pairs of links until it converges. In [48] it was proven that such Metropolis algorithm converges to the Gibbs measure $e^{\mu N_{4}}$ in the equilibrium ensemble of random undirected ER networks with fixed vertex degree or RRG.

In [11] it has been shown that, given the bond formation probability, $p$, in the initial graph in constrained ER graph or in RRG, the evolving network above the critical value of chemical potential for triangles $\mu_{3}$ splits into the maximally possible number of clusters, $N_{c l}$ :

$$
N_{c l}=\left.\left[\frac{N}{N p+1}\right]\right|_{N \gg 1} \approx\left[\frac{1}{p}\right]
$$

where $[x]$ means the integer part of $x$ and the denominator $(N p+1)$ defines the minimal size of formed cliques. The asymptotic limit $\sim\left[p^{-1}\right]$ at $N \rightarrow \infty$ in (4) is independent on the particular set of corresponding vertex degrees, $\left\{v_{1}, \ldots, v_{N}\right\}$. For RRG with degree $d$ the number of clusters 
tends to $[N /(d+1)]$. It has been shown in [11] that clustering of evolving constrained Erdős-Renyi network or RRG under condition of three-cycle maximization, occurs as a first order phase transition where $\mu_{3}$ is a control parameter.

\section{B. Clusterization of the perturbed RRG}

Consider the RRG with degree $d$ perturbed by the $\mu_{4}$ term

$$
H=\mu_{4} \operatorname{Tr} A^{4}
$$

and vary the chemical potential. At some $\mu_{4, \mathrm{cr}}^{\mathrm{RR}}$ the network gets rearranged into bipartite phase with the fixed number of weakly interacting clusters. The corresponding adjacency matrix emerged upon transition is presented at Fig. 1. All bipartite clusters are almost maximal and have the same size. The similar phase transition takes place for the constrained ER network however it that case the size of bipartite clusters has nontrivial distribution.

The spectrum of the adjacency matrix $A$ and the Laplacian matrix $L=D-A$ where $D=\operatorname{diag}\left(d_{1}, \ldots, d_{n}\right)$ is encoded in the spectral density defined as

$$
\rho(\lambda)=\left\langle\sum_{i} \delta\left(\lambda-\lambda_{i}\right)\right\rangle_{\mathrm{RRG}}, \quad A v_{i}=\lambda_{i} v_{i}
$$

where the averaging over ensemble is assumed. The unperturbed spectral density of RRG obeys the KestenMcKay distribution

$$
\rho(\lambda)=\frac{\sqrt{4(d-1)-\lambda^{2}}}{2 \pi\left(d^{2}-\lambda^{2}\right)} .
$$

For the RRG a spectral density of the graph Laplacian coincides with the shifted spectral density of adjacency matrix and we can use both of them as the Hamiltonian of a particle propagating on the RRG. The spectral density gets modified upon transition and acquires the following structure. The central band corresponds to a continuum spectrum. If there would be no intercluster interaction, then there would be degenerate states symmetric with respect to the central band, and the degeneracy of eigenvalues would correspond to the number of eigenvalue instantons. We can interpret these modes as the bound state of the particle localized at the cluster. However due to the intercluster interaction we get two nonperturbative bands instead of the degenerate bound states. For each realization pair of isolated eigenvalues corresponding to the bipartite cluster symmetrically tunnel from the perturbative band in the opposite directions. The evolution of spectral density of the adjacency matrix is presented at Fig. 2.

We have also considered the evolution of the spectral density for the constrained ER network. The clusterization

(a)

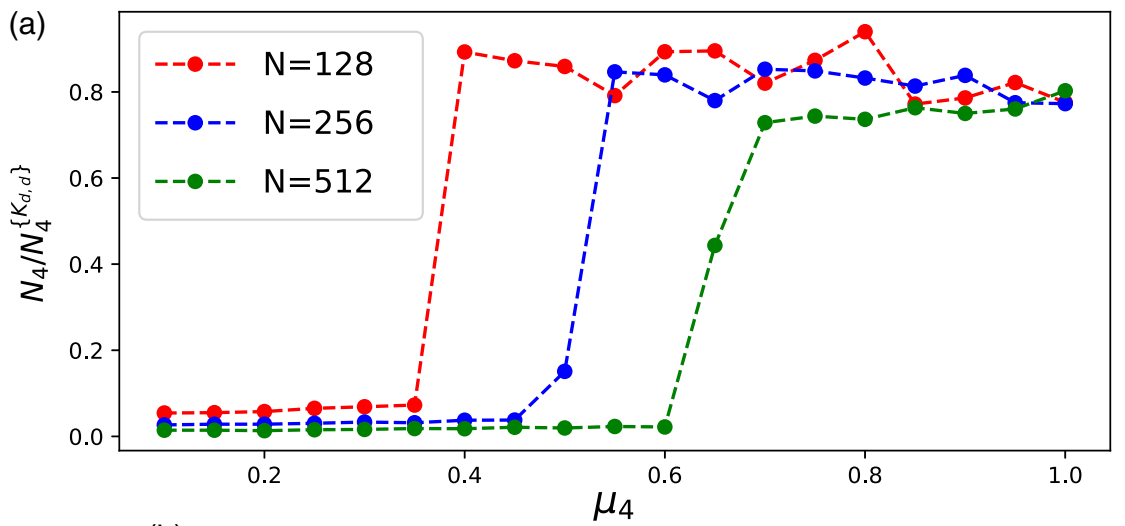

(b)
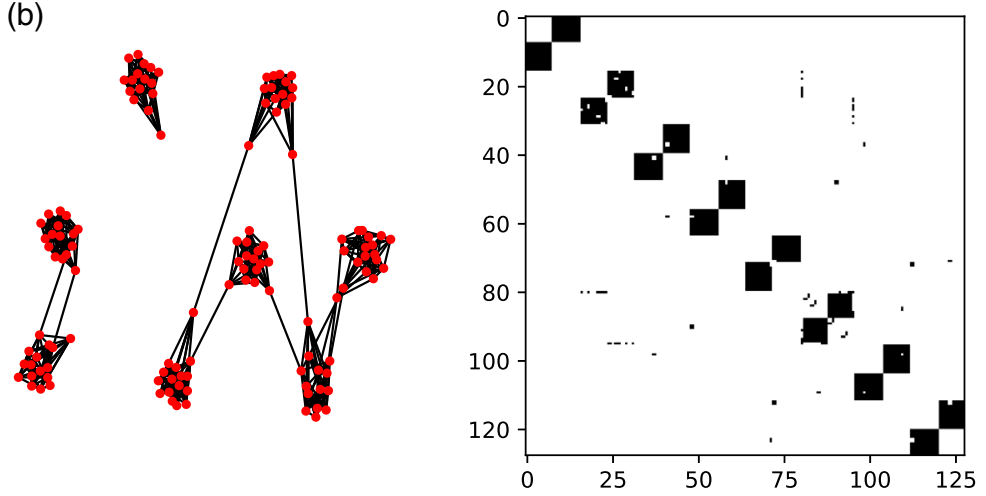

FIG. 1. (a) The dependence of number of $C_{4}$ on the chemical potential $\mu_{4}$ for RRG of different sizes and $d=8$. (b) Ground state and its adjacency matrix at critical $\mu_{4}$. 


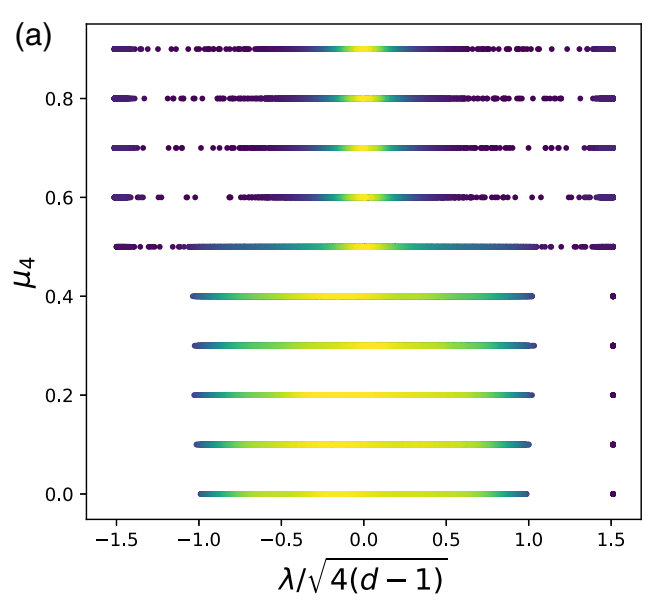

(b)

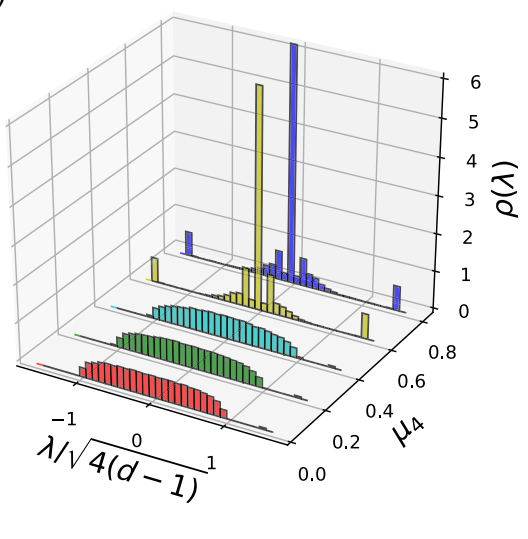

FIG. 2. The dependence of the spectral density of adjacency matrix on the chemical potential $\mu_{4}$ for RRG of $N=256, d=8$.

pattern into bipartite clusters is the same with some peculiarities. In this case the sizes of the bipartite clusters are different with some size distribution. The spectral density of graph Laplacian acquires the three-band structure with more wide nonperturbative bands.

Note that in the spectrum of the graph Laplacian half of eigenvalues corresponding to instantons $\lambda_{i,+}$ are the soft modes while half of eigenvalues are the hard modes $\lambda_{i,-}$. All clusters are bipartite hence there is specific interaction inside each bipartite cluster that connects different scales corresponding to eigenvalues $\lambda_{i,-}, \lambda_{i,+}$. It provides the entanglement of the bipartite state.

The symmetry breaking pattern at the phase transition is $S O(2 N) \rightarrow S O\left(\frac{N}{d}\right) \times S O\left(\frac{N}{d}\right)^{d}$. Let us emphasize that a formation of the bipartite clusters can be observed in the real-time during the Metropolis cooling. The formation and evolution of the cluster occurs simultaneously with the evolution of pair of isolated eigenvalues.

\section{RRG with hard-core constraint and combinatorial quantum gravity}

Let us consider now CRRG and argue that upon the phase transition the different ground state emerges. If only hard-core constraint is imposed the ground state involves the ensemble of noninteracting or weakly interacting hypercubes and a separate bipartite closed ribbon. However if additional connectedness condition is imposed, then the ground state will be identified as the closed chain of weakly interacting hypercubes.

The hard-core constraint was introduced in the interesting model of combinatorial quantum gravity suggested in [21], which is based on the combinatorial graph curvature invented by Ollivier [22]. The Ollivier curvature is quite involved for generic graph however it can be simplified for special types of the graph ensembles. It was suggested in [21] to consider as the simple model for combinatorial quantum gravity the ensemble of RRG with two additional constraints. First, the bipartiteness condition is imposed, and second it is assumed that two squares can have only one common link, which to some extent can be considered as the analog of the hard-core condition for extended objects. It was shown recently [24] that for the large class of graphs the Ollivier curvature gets reduced to the Ricci curvature in the thermodynamic limit.

Under these conditions the density of Ollivier curvature in the mean field approximation for the CRRG acquires the simple form

$$
R_{i j}=-\frac{1}{d}\left[(2 d-2)-N_{4}(i j)\right],
$$

where $N_{4}(i j)$ is the number of squares supported at the $(i j)$ edge. Then one introduces the analog of the Ricci scalar

$$
R=\sum_{i} \sum_{j} R(i j)
$$

which for the CRRG ensemble gets reduced to

$$
R_{\mathrm{CRRG}}=-\frac{8}{d}\left[\frac{d(d-1)}{2} N-N_{4}\right],
$$

where $N_{4}$ is the total number of $C_{4}$.

Therefore for CRRG the partition function involving the Hamiltonian $H=\mu_{4} T r A^{4}$ acquires the meaning of the combinatorial version for the action of Euclidean Einstein gravity in mean field approximation

$$
Z_{\text {comb }}=\int d g \exp \left(\alpha \int \sqrt{g} R\right),
$$

where the integral over the metric comes from the summation over the CRRG ensemble. The chemical potential for $C_{4}$ defines the gravitational coupling constant upon some rescaling 

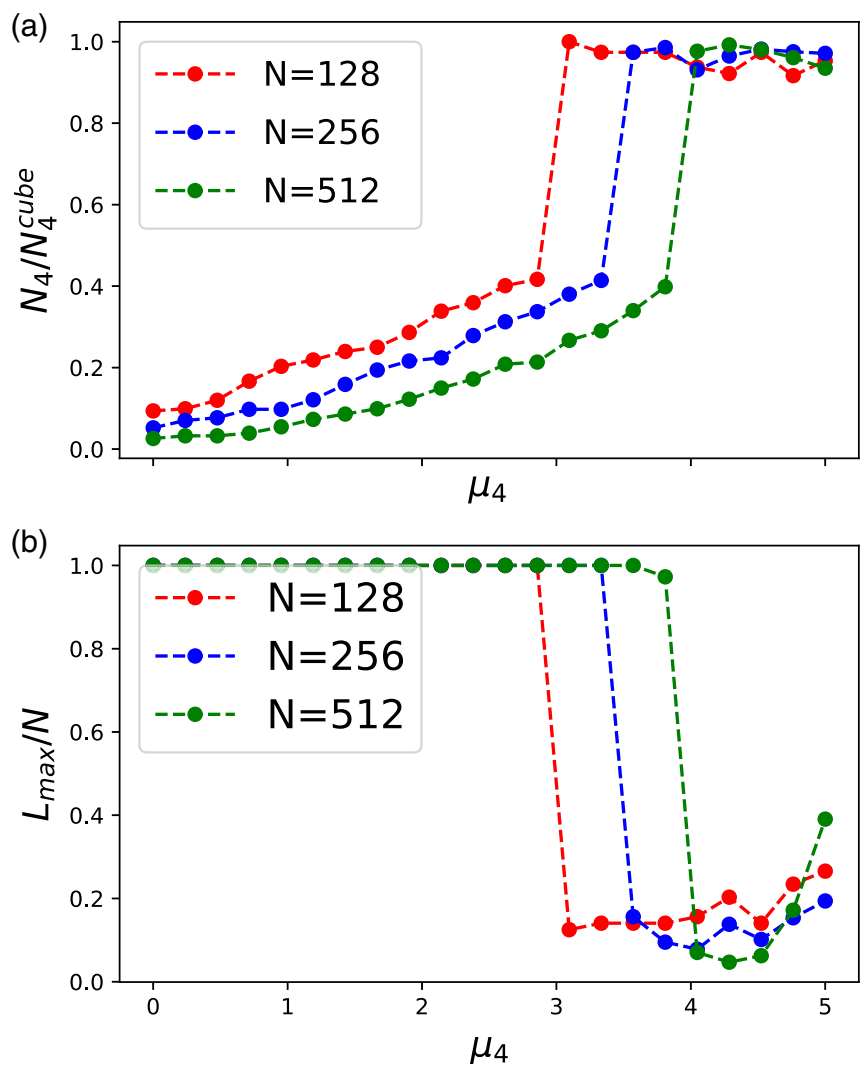

FIG. 3. (a) The number of $C_{4}$ in dependence on the parameter $\mu_{4}$ in CRRG of $d=4$ and different size. (b) The portion of the nodes in the bipartite closed ribbon in dependence on the parameter $\mu_{4}$. $\alpha \propto \mu_{4}$

We have investigated numerically the phase transition for CRRG for different degrees $d$. First of all, we have found that the first order phase transition takes place at $\mu_{4}^{\mathrm{CRRG}}$ for $d>3$. We have observed a set of isolated or weakly connected $d$-dimensional hypercubes at $\mu=\mu_{4}^{\mathrm{CRRG}}$, the number of hypercubes can be estimated as

$$
N_{\text {cube }}=\left.\left[\frac{N}{2^{d}}\right]\right|_{N \gg 1} .
$$

Numerical results for the number of $C_{4}$ are presented in Fig. 3, there is a explicit hysteresis typical for phase transitions of first order. Second, we observe that if there is no enough nodes to form one more hypercube and for $\mu_{4}>\mu_{4}^{\mathrm{CRRG}}$ part of nodes are organized in the closed bipartite ribbon similar to one discussed in [26]. The number of the nodes in the closed ribbon depends on $\mu_{4}$ nontrivially, see Fig. 3(b). The typical configurations in clusterized CRRG for different values $d$ and $\mu$ are presented at Fig. 4. The typical configurations in clusterized CRRG for different values $\mathrm{d}$ and $\mu$ are presented at Figs. 4 and 5 .

Next, we impose the second connectedness constraint in CRRG and simulated CRRG with this additional restriction. We have observed the phase transition for $d=3$ with the bipartite ribbon as the ground state confirming the result of [26]. However, for $d>3$ there is a clear-cut first order phase transition (see Fig. 6) with the formation of the
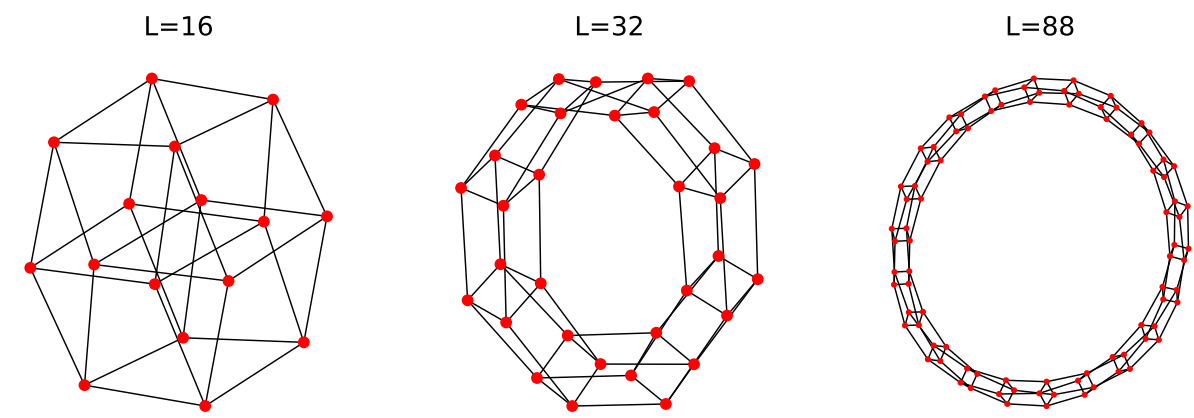

FIG. 4. Typical subgraph configurations in the clustered phase involving hypercubes and composite bipartite strings.
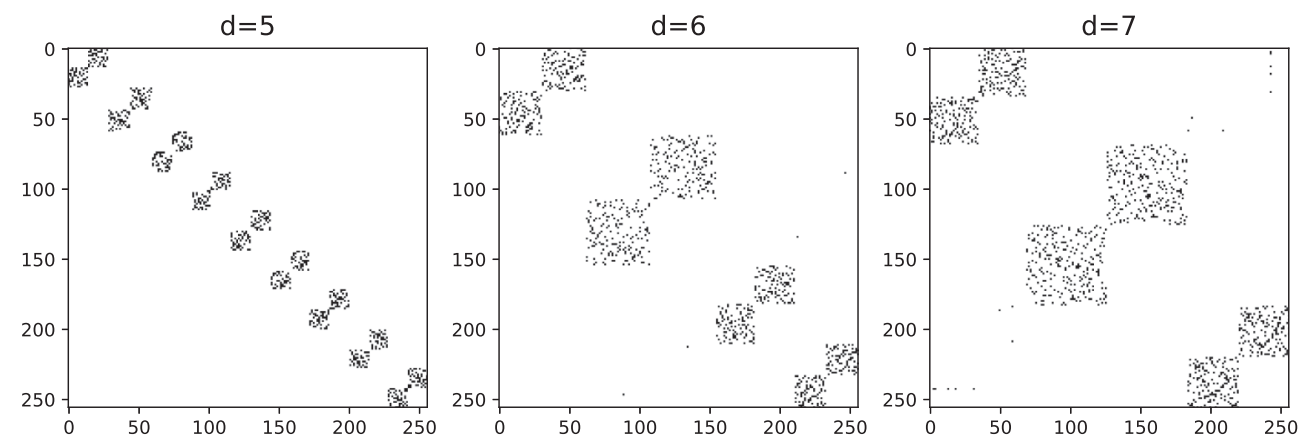

FIG. 5. Typical adjacency matrices in the clustered phase for RRG of $N=256$ and different values of $d$. 

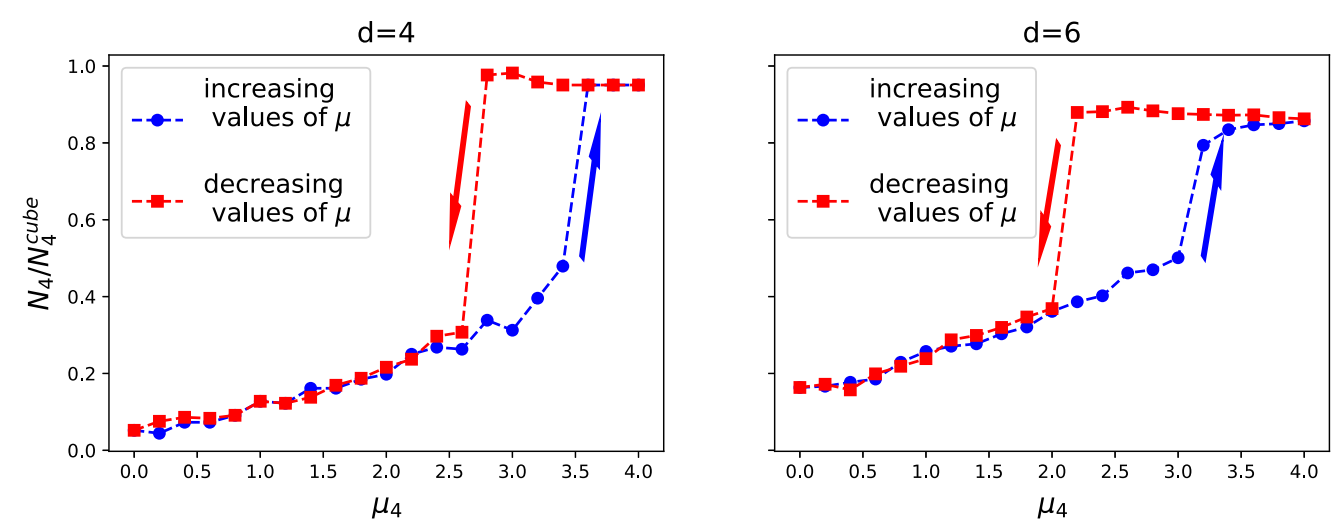

FIG. 6. Hysteresis in dependence of the number of $C_{4}$ for RRG of $N=256$ and different degrees.
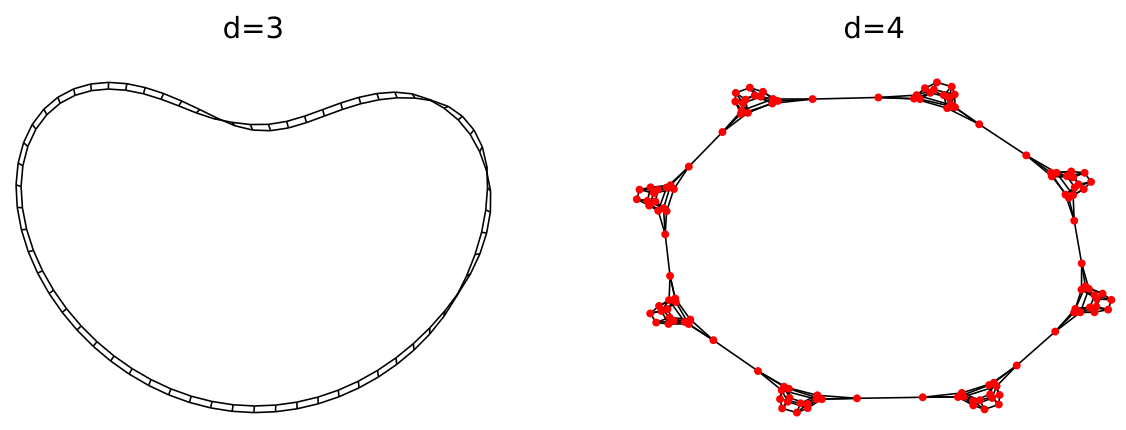

$d=5$

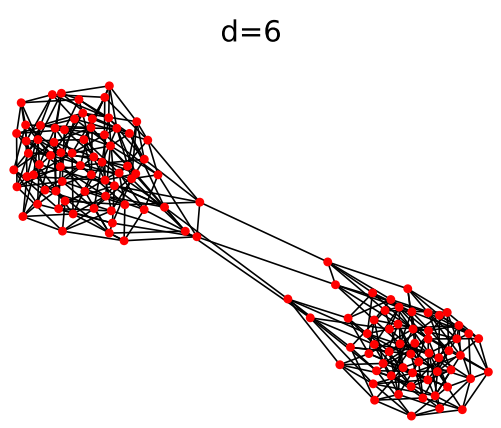

FIG. 7. Ground state of CRRG with connectedness constraint and different degree $d$ for RRG of $N=128$.

clusterized ground state.. It is identified as closed chain of weakly connected hypercubes (Fig. 7) and all $C_{4}$ belong to hypercubes.

\section{INTERACTING THERMOFIELD DOUBLES AND HOLOGRAPHIC DUAL}

\section{A. Towards the holographic dual for hypercube phase}

We have argued above that at the transition point the RRG network with hard-core constraint gets disintegrated into the ensemble of noninteracting or weakly interacting hypercubes with additional closed bipartite ribbon. If the connectedness condition is added the emerging configuration is identified as the single bipartite ribbon for $d=3$ and the closed chain of weakly connected hypercubes for $d>3$. In this study we are guided by the analogy between the exponential random graphs and matrix models. Hence we could question if a kind of holographic representation of our partition function for the graph ensemble can be developed somewhat similar to the matrix model representation of Jackiw-Teitelboim (JT) gravity $[49,50]$. In that case the $(0+1)$ boundary of Euclidean quantum mechanics is presented holographically by JT gravity. More precisely the discussion in [50] suggest that the bulk gravity is dual to the ensemble of boundary theories.

Before proceeding further two remarks are in order. First, we shall try to develop the holographic picture for the clusterized phase only for CRRG and CRRG with connectedness constraint. Second, we are guided by the idea that quantum entanglement of the boundaries can lead to 
the classical geometry in the bulk [41]. The first clear-cut of such situation has been developed in [40] for the eternal black hole with two boundaries. In the Euclidean version the corresponding geometry with several boundaries is provided by the multiboundary wormholes [44]. The boundary components can be entangled via the gravitational bulk. The recent discussion concerning this scenario can be found in $[42,43]$. We shall have in mind this pattern of holography in what follows.

To develop holographic interpretation we should clarify what is the meaning of hypercubes and bipartite ribbons in the gravity framework. First take a look at the hypercubes. It was noted in [37] that the hypercube adjacency matrix $A_{\text {hyper }}$ exactly coincides with the interaction term in the Maldacena-Qi model [38] for the near $\mathrm{AdS}_{2}$ gravity.

$$
A_{\text {hyper }}=H_{\mathrm{int}}=i \sum_{k} \gamma_{k}^{L} \gamma_{k}^{R} \text {. }
$$

The mapping is going as follows. Let consider the tensor product representation for the hypercube Hamiltonian

$$
H_{d}=\sigma_{0} \otimes H_{d-1}+\sigma_{1} \otimes \overbrace{\sigma_{0} \otimes \cdots \otimes \sigma_{0}}^{d-1}, \quad H_{1}=\sigma_{1},
$$

and define the $\gamma$ matrices representing Majorana fermions as

$$
\begin{aligned}
& \gamma_{k}^{L}=\overbrace{\sigma_{1} \otimes \cdots \otimes \sigma_{1}}^{k-1} \otimes \sigma_{3} \otimes \overbrace{\sigma_{0} \otimes \cdots \otimes \sigma_{0}}^{d-k}, \\
& \gamma_{k}^{R}=\overbrace{\sigma_{1} \otimes \cdots \otimes \sigma_{1}}^{k-1} \otimes \sigma_{2} \otimes \overbrace{\sigma_{0} \otimes \cdots \otimes \sigma_{0}}^{d-k},
\end{aligned}
$$

then the Hamiltonian can be written as

$$
A_{\text {hyper }}=i \sum_{k=1}^{d} \gamma_{k}^{L} \gamma_{k}^{R}
$$

Since we are looking at the RRG ensemble, the spectrum of hypercube adjacency matrix that reads as

$$
E_{n}=-d+2 n, n=0 \ldots d \quad \operatorname{deg}\left(E_{n}\right)=C_{n}^{d}
$$

coincides with the shifted spectrum of the hypercube graph Laplacian $L=d I-A$ where $I$ is identity matrix. Therefore the one-particle Hamiltonian of a free particle on the hypercube gets mapped into the interaction term of Maldacena-Qi Hamiltonian for the Majorana fermions.

On the other hand is was found in $[37,39]$ that the ground state of the Maldacena-Qi model at strong coupling limit is well approximated by the TFD at $\beta=0$ in boundary quantum mechanics of Majorana fermions

$$
|\mathrm{TFD}\rangle=\sum_{n} e^{-\beta E_{n}}|n\rangle_{L}|n\rangle_{R}
$$

At large $N$ boundary degrees of freedom in each component of TFD are degrees of freedom forming the eternal black hole in anti-de Sitter (AdS) [40] or in Minkowski space or wormhole in the Euclidean space. If there are several entangled components the gravity dual is the multiboundary wormhole.

Now let us turn to our proposal for the gravity dual of the clusterized phase. For CRRG we have set of noninteracting or weakly interacting hypercubes. Each hypercube corresponds to the TFD of entangled $d$ Majorana fermions. If $d$ is large it is natural to assume that each TFD yields the path in the dual geometry moreover all TDFs are entangled yielding the geometry of nontraversable multiboundary wormhole in AdS space somewhat analogously to the arguments in [42] if we have noninteracting but entangled hypercubes.

If $d$ is small we still have the TFDs of $d$ Majorana fermions forming the Majorana dimers. It is however impossible to assume that such TDF corresponds to the fragment of the gravity dual since it has not enough degrees of freedom. On the other hand the total number of TDFs is large so a kind of holography can be expected nevertheless. We can use the arguments recently suggested in the holographic representation of the error-correcting codes [45]. It was argued in [45] that boundary Majorana dimers through their entanglement yield the geodesics in the bulk geometry allowing its partial restoration. However contrary to [45] here we have TFDs for the groups of $d$ Majorana dimers.

When the connectedness constraint is added we get the closed chain of hypercubes and therefore the closed chain of TDFs as a ground state. However now we certainly have the interaction between TDFs. Following arguments from $[42,43]$, we conjecture that the bulk dual is the traversable multiboundary wormhole if $d$ is large enough. At small $d$ we can apply the similar logic based on the error-correcting codes. Here we have the interaction between the entangled groups of Majorana dimers.

Let us complete this section with a short remark concerning the topology of the emerging hypercube phase. The isolated $n$ hypercube enjoys the hyperoctahedral symmetry group $C_{n}$, which is the wreath product of $S_{2}$ and $S_{n}$ where $S_{n}$ is the symmetric group of degree $n$. It has nontrivial first and second homology groups $H_{1}\left(C_{n}, Z\right)=(Z / 2)^{2}, n \geq 2$, $H_{2}\left(C_{n}, Z\right)=(Z / 2)^{3}, n \geq 4$. This allows us to look for the topologically stable closed chain of hypercubes.

\section{B. D0-branes quantum mechanics and black hole formation}

Matrix models provide the framework for a formation of extended objects from ensemble of D0 branes [51]. Above we have made the proposal concerning the holographic 
interpretation of the hypercube phase of perturbed CRRG. Let us make a short comment on the somewhat analogous situation in the holographic interpretation of a matrix model when the typical matrix acquires the block-diagonal form.

According to holography any transition in the ground state in the boundary matrix model gets mapped into some transition in the dual bulk theory. The transitions can be identified via the changes of the spectral density and the corresponding form of matrix analogously to our discussion of exponential random graphs. It was suggested in the context of the large $N(1+1)$ SYM theory on the circle that the distribution of the eigenvalues of Wilson loops plays the key role. The flat distribution holographically corresponds to a black string while the gaped distribution corresponds to a black hole [52].

Somewhat similar classification has been used in the context of the deconfinement phase transition [46,53-55]. It was argued that again there are three phases: confined, partially deconfined and completely deconfined in the boundary theory. The typical matrix corresponding to the partially deconfined phase has deconfined $M \times M$ block filled densely, which presumably corresponds to the small black hole in the dual bulk while the rest $S U(N-M)$ sector corresponds to the black hole exterior. The entropy in this phase has intermediate form $S=\epsilon N^{2}$, where $\epsilon \ll 1$. The number of blocks in the typical matrix corresponds to the number of small black holes.

Having the holographic picture in mind we can speculate on the analogy of the phases in the deconfinement transition with the phases in the exponential random graphs. In the ER network enriched by three cycles the single dense cluster gets emerged [6], which presumably holographically corresponds to the small black hole. The RRG perturbed by three cycles develops several interacting dense clusters [11], which presumably correspond to the several interacting small black holes similarly to $[54,55]$.

\section{RRG AS THE MODEL FOR A HILBERT SPACE AND LOCALIZATION}

The RRG is now the popular model for a investigation of the localization phenomena in a interacting many-body system. It models the Hilbert space of a model and the MBL in the physical space gets mapped into one-body localization at RRG following the initial arguments suggested in [27]. The Laplacian of the graph plays the role of the Hamiltonian for the propagating degree of freedom. If the wave function of an effective one-particle system in the Fock space is close to the state of the initial many-body system, then the localization in the Fock space occurs. Hence, one can identify the localized state in the Fock space with the particle in the initial many-body system. Meanwhile, if the wave function of the effective oneparticle system in the Fock space is expanded over a large number of states of the many-body system, this regime is understood as a delocalized in the Fock space. Similarly to the localization in the real space, the notion of a mobility edge can be introduced in the Fock space as well.

Technically one can analyze the ergodic properties through the fractal dimensions $D_{q}$, which show the spread of a state in the Fock space or via level spacing distribution. Ergodic states at infinite temperatures are spread homogeneously over the entire Fock space hence $D_{q}=1$. Instead, nonergodic states cover only a vanishing fraction of the Fock space, $0<D_{q}<1$, while a localization in the Fock space requires $D_{q}=0$. Hence in terms of the Fock space the MBL transition can be identified as transition from $D_{q}=1$ in the ergodic space phase to $D_{q}<1$ in the MBL phase. Different aspects of the one-body localization on RRG with diagonal disorder have been discussed in [31-34,36]. Recently some arguments concerning the possible Kosterlitz-Thouless nature of this transition in terms of the Fock space have been developed in [56].

It was argued in $[57,58]$ that the so-called scars in the real space could provide the MBL phase. They were argued to be related to the peculiar fragmentation of the Fock space in the many-body system. Generically it is expected that the disorder is the source of the Hilbert space fragmentation; however, there are clear examples $[59,60]$ showing that the local conservation laws also can play such role. In particular it was shown in $[59,60]$ that the combination of the charge and dipole number conservation amounts to the fragmentation of the Fock space into the exponentially many scars supporting localized states. In this case there is no need for a disorder. More formal group theory based arguments concerning the fragmentation of the Hilbert space can be found in [61,62]. The use of the Krylov basis for the fragmentation phenomenon has been discussed in [63].

It was found in [30] that the RRG perturbed by the chemical potential for three cycles $\mu_{3}$ gets defragmented into the fixed number of weakly coupled clusters at come critical $\mu_{3 \text {,crit }}$. The spectrum of graph Laplacian acquires the two-band structure, the number of the eigenvalues in the nonperturbative band is equal to the number of clusters and the eigenvectors in the nonperturbative band are localized. Hence we have explicit example of the localization in the Fock space via its fragmentation. One could question the underlying mechanism for the fragmentation in this case similar to the hidden conservation laws in other examples. The fixed degrees of the nodes in the RRG play the role of the local conservation laws. To clarify this point we have considered the constrained ER with the degree conservation constraint. The fragmentation of the network similar to the RRG takes place; however, if we drop off the degree conservation constraint, then the pure ER network does not exhibit the fragmentation phase transition. Note that the degree conservation constraint in the Fock space corresponds to the nonlocal constraint in the real space.

The identification of the graph responsible for the Hilbert space of particular interacting many-body system is not a 
simple task. The nodes correspond to the states while the links connect the states related by the resonant conditions. The cycles have the meaning of the higher resonances [64] for instance the three cycle discussed in [30] corresponds to the three nodes whose energies are organized in such way that the interaction provides the resonant conditions for all pairs of states. In this study we have considered the $C_{4}$ that correspond to the resonant conditions for four states. We have demonstrated that the increasing of the number of four resonances yields the structural disorder in the graph upon the phase transition via the network fragmentation.

We look at the localization of the modes in the RRG and CRRG in hypercube phase similarly to [30]. Preliminary study shows that, similarly to [30], the modes corresponding to the bipartite clusters in RRG are localized in agreement with the naïve expectations. The pure hypercube corresponds to the integrable system hence it enjoys the localized modes. However when the interaction between the hypercubes is switched on it can be seen that there is localization in the whole spectrum. The question concerning the existence of mobility edge in this case need for the additional study.

Note that the ER network with chemical potential for $C_{4}$ without degree conservation constraint can be considered as well. In this case the analytical analysis is available and it perfectly fits with the numerical simulations [65]. In this case the fragmentation of the Hilbert space does not take place and all modes are delocalized. The ER network has the intrinsic disorder in the graph Laplacian due to the degree distribution however it does not lead to localization.

We do not know exactly what physical system has the Hilbert space which we are treating as the perturbed RRG. However a few remarks are in order. First note that the perturbation by $C_{4}$ amounts to the emerging $Z_{2}$ symmetry in the Hilbert space. Secondly one could question if some physical system has the hypercube as the Hilbert space. Some indication of the relevance of the single hypercube to the Hilbert space of spin chain has been noted in [56], but this point certainly needs further analysis.

Finally note that the Fock space perspective provides the interesting twist of the holographic picture. Now we consider the bulk holographic dual not for representation of the boundary partition function via the path integral over fields but via sum over the Hilbert space represented by some ensemble of graphs. In particular the combinatorial quantum gravity we have discussed above acquires the meaning of the effective gravity in the Hilbert space where the chemical potential for the four resonances plays the role of the gravitational coupling. The Ollivier curvature plays the role of the discrete Ricci curvature in the Hilbert space. The multiboundary wormhole interpretation of the hypercube phase we suggested above has the interpretation of the dual bulk geometry for the boundary Hilbert space ensemble for some theory that we do not identify.

\section{DISCUSSION}

In this study we investigated numerically the nonperturbative phenomena in exponential random graphs with the degree conservation constraints. They are similar to the eigenvalue instantons in matrix models however the degree conservation constraint corresponds to the account of the non-singlet sector in matrix model context. It was argued that the degree conservation induces the first order phase transition of a network into bipartite clusters at $\mu_{4}>\mu_{4}^{c}$ both for RRG and constrained ER networks. The induced network bipartiteness at strong coupling phase is the dynamical phenomenon.

The RRG with $C_{4}$ chemical potential has been used as the model for the mean field version of combinatorial quantum gravity if the additional hard-core constraint is imposed. We have performed the detailed numerical analysis of CRRG. It turned out that the first order phase transition with a clear-cut hysteresis takes place at $\mu_{4}^{C R R G}$ for $d>3$. Nearby the phase transition point the CRRG decays into the ensemble of separated noninteracting or weakly interacting hypercubes supplemented with closed bipartite ribbon. Such constituents of the clustered phase, hypercubes and bipartite ribbon, have appeared in all numerical experiments at different $d$. If we impose the additional graph connectedness condition for CRRG, then a structure of the clusterized phase gets changed and depends on value of $d$. At $d=3$ there is a phase transition with the formation of the single bipartite closed ribbon in agreement with [26]. The hard core constraint does not prevent from the clusteriztion in the mean field approximation and for $d>3$ there is the first order phase transition with clear-cut hysteresis and formation of the weakly connected chain of hypercubes.

Using the relation between the hypercubes and TFDs, we have made the proposal concerning the holographic interpretation of the bipartite hypercube phase. Namely we have conjectured that the ground state of CRRG at large $d$ holographically corresponds to the multiboundary nontraversable wormholes where the number of boundaries coincides with the number of isolated hypercubes. At small $d$, when there are no enough degrees of freedom to form a wormhole, we have assumed the relation with a holographic representation of the error-correcting codes in terms the Majorana dimers [45]. The multiboundary wormhole is supplemented with the bipartite closed ribbon, which is related to the specific moduli spaces of the mapping of the Riemann surfaces into the sphere with three branching points [66] and presumably deals with the topological membrane. If we add the connectedness constraint for CRRG the suggested holographic dual for the closed chain of weakly interacting hypercubes presumably is the multiboundary traversable Euclidean wormhole. It would be interesting to relate this proposal with the tensor network representation of AdS geometry [67] based on the entanglement 
renormalization. Certainly the holographic dual of the bipartite hypercube phase deserves a further study.

Our study demonstrates that nonperturbative phenomena in exponential random graphs are more tractable for numerical simulations than in the matrix models. This allows us to visualize the eigenvalue instantons as the process of the creation of the corresponding bipartite clusters. In the context of the hypercube phase it is also possible numerically to fix $\mu_{4}$ and increase $N$, this procedure allows to consider the finite-size effects. Such analysis in a two-star model [18] provides the clear picture for the evaporation of the single cluster. In our case the similar process of a evaporation of the hypercubes can be expected. It is natural since $N^{-1}$ is the effective coupling. Since, according to our proposal, the hypercube at large $d$ being the TFD state is holographically dual to the wormhole, then the evaporation of hypercube presumably can be related to a kind of wormhole evaporation. We hope to discuss this point elsewhere.

Treating RRG as a model for the Hilbert space of manybody system we indicated a few new patterns of the Hilbert space fragmentation induced by the higher resonances. The fragmentation of the Hilbert space is related to the MBL phase in the real space and the preliminary study indicates the oneparticle localization of the cluster modes occurs indeed. We shall present the more detailed analysis of the inverse participation ratio (IPR), level spacing distribution, and the entanglement entropy in the hypercube phase elsewhere. It would be also interesting to consider the RRG supplemented with the fluxes, which would yield the Parisi hypercube model upon fragmentation of the Hilbert space. The consideration of the SYK model on the perturbed RRG along the logic of [68] could be of some importance as well.

One more interesting development concerns the dual holographic picture for the Hilbert space in the spirit of the operator-state correspondence. The fragmentation of the
Hilbert space in the hypercube phase provides the firm starting point along this line having in mind the wormhole interpretation of the hypercube ground state.

Our study also provides some suggestions concerning the partial deconfinement scenario. In the holographic context, the block diagonal form of the matrix presumably corresponds to the partial deconfinement and formation of the small black holes in the bulk. The spectral density of the Wilson or Polyakov loops acquires the gaps. Our study suggests that the correspondence can be more general and some version of wormholes can be relevant. They correspond to the bipartite block diagonal matrices and the spectral density with two gaps. It would be interesting to develop these arguments further.

Finally, we remark that the bipartite large $N$ ensemble arises naturally in low energy QCD when the integration over instanton moduli spaces is mimicked by the matrix model with a simple potential [69]. The degree conservation condition should be implemented in this model by hand since the degree of the node is a topological invariant. Our study suggests that when the quartic term in the potential starts to dominate in the matrix measure one could expect that the instanton fluid phase gets transformed into the instanton molecular phase when each "molecule" corresponds to the bipartite cluster.

\section{ACKNOWLEDGMENTS}

We are grateful to I. Khaimovich, V. Kravtsov, A. MIlekhin, and S. Nechaev for the useful discussions. A. G. acknowledges Grant No. 075-015-2020-801 of Ministry of Science and Higher Education of Russian Federation. The work of O. V. is supported within the framework of the Academic Fund Program at the HSE University in 2020/2021 (Grant No. 20-01-041).
[1] P. H. Ginsparg and G. W. Moore, Lectures on 2-D gravity and 2-D string theory, https://arxiv.org/abs/hep-th/9304011 arXiv:hep-th/9304011.

[2] S. H. Shenker, The strength of nonperturbative effects in string theory, in The Large N Expansion in Quantum Field Theory and Statistical Physics (1993), pp. 809-819, https:// doi.org/10.1142/9789814365802_0057.

[3] F. David, Nonperturbative effects in matrix models and vacua of two-dimensional gravity, Phys. Lett. B 302, 403 (1993).

[4] M. Marino, Lectures on non-perturbative effects in large $\mathrm{N}$ gauge theories, matrix models and strings, Fortschr. Phys. 62, 455 (2014).
[5] J. Ambjorn, R. Loll, Y. Watabiki, W. Westra, and S. Zohren, New aspects of two-dimensional quantum gravity, Acta Phys. Pol. B 40, 3479 (2009), https://arxiv.org/abs/0911 .4208 .

[6] D. Strauss, On a general class of models for interaction, SIAM Rev. 28, 513 (1986).

[7] Z. Burda, J. Jurkiewicz, and A. Krzywicki, Network transitivity and matrix models, Phys. Rev. E 69, 026106 (2004).

[8] J. Park and M. E. J. Newman, Solution for the properties of a clustered network, Phys. Rev. E 72, 026136 (2005).

[9] J. Park and M. E. J. Newman, Solution of a 2-star model of a network, Phys. Rev. E 70, 066146 (2004). 
[10] A. Annibale and O. Courtney, The two-star model: Exact solution in the sparse regime and condensation transition, J. Phys. A 48, 365001 (2015).

[11] V. Avetisov, M. Hovhannisyan, A. Gorsky, S. Nechaev, M. Tamm, and O. Valba, Eigenvalue tunnelling and decay of quenched random networks, Phys. Rev. E 94, 062313 (2016).

[12] S. Chatterjee and P. Diaconis, Estimating and understanding exponential random graph models, Ann. Stat. 41, 2428 (2013).

[13] C. Radin and M. Yin, Phase transitions in exponential random graphs, Ann. Appl. Probab. 23, 2458 (2013).

[14] C. Radin and L. Sadun, Phase transitions in a complex network, J. Phys. A 46, 305002 (2013).

[15] E. Guzman-Gonzalez, Isaac Perez Castillo, and F. L. Metz, Phase transitions in atypical systems induced by a condensation transition on graphs, Phys. Rev. E 101, 012133 (2020).

[16] F. A. Lopez and A. C. C. Coolen, Imaginary replica analysis of loopy regular random graphs, J. Phys. A 53, 065002 (2020).

[17] F. A. Lopez and A. C. C. Coolen, Transitions in loopy random graphs with fixed degrees and arbitrary degree distributions arXiv:2008.11002.

[18] A. Gorsky and O. Valba, Finite-size effects in exponential random graphs, J. Complex Netw. 8, 1 (2020).

[19] D. Foster, J. Foster, M. Paczuski, and P. Grassberger, Communities, clustering phase transitions, and hysteresis: Pitfalls in constructing network ensembles, Phys. Rev. E 81, 046115 (2010).

[20] M. Tamm, A. Shkarin, V. Avetisov, O. Valba, and S. Nechaev, Islands of Stability in Motif Distributions of Random Networks, Phys. Rev. Lett. 113, 095701 (2014).

[21] C. A. Trugenberger, Combinatorial quantum gravity: Geometry from random bits, J. High Energy Phys. 09 (2017) 045.

[22] Y. Ollivier, Ricci curvature of Markov chains on metric spaces, J. Funct. Anal. 256, 810 (2009).

[23] Y. Ollivier, A survey of Ricci curvature for metric spaces and Markov chains, Adv. Stud. Pure Math. 57, 343 (2010).

[24] P. van der Hoorn, W. J. Cunningham, G. Lippner, C. Trugenberger, and D. Krioukov, Ollivier-Ricci curvature convergence in random geometric graphs, Phys. Rev. Research 3, 013211 (2021).

[25] C. Kelly, C. Trugenberger, and F. Biancalana, Emergence of the circle in a statistical model of random cubic graphs, Classical Quantum Gravity 38, 075008 (2021).

[26] C. Kelly, C. Trugenberger, and F. Biancalana, Self-assembly of geometric space from random graphs, Classical Quantum Gravity 36, 125012 (2019).

[27] B. L. Altshuler, Y. Gefen, A. Kamenev, and L. S. Levitov, Quasiparticle Lifetime in a Finite System: A Nonperturbative Approach, Phys. Rev. Lett. 78, 2803 (1997).

[28] D. Basko, I. Aleiner, and B. Altshuler, Metalâ insulator transition in a weakly interacting many-electron system with localized single-particle states, Ann. Phys. (Amsterdam) 321, 1126 (2006).

[29] I. V. Gornyi, A. D. Mirlin, and D. G. Polyakov, Dephasing and Weak Localization in Disordered Luttinger Liquid, Phys. Rev. Lett. 95, 046404 (2005).
[30] V. Avetisov, A. Gorsky, S. Nechaev, and O. Valba, Localization and non-ergodicity in clustered random networks, J. Complex Netw. 8, 1 (2020).

[31] A. De Luca, B. Altshuler, V. Kravtsov, and A. Scardicchio, Anderson Localization on the Bethe Lattice: Nonergodicity of Extended States, Phys. Rev. Lett. 113, 046806 (2014).

[32] V. E. Kravtsov, B. L. Altshuler, and L. B. Ioffe, Nonergodic delocalized phase in Anderson model on Bethe lattice and regular graph, Ann. Phys. (Amsterdam) 389, 148 (2018).

[33] K. S. Tikhonov and A. D. Mirlin, Fractality of wave functions on a Cayley tree: Diï $\neg$ erence between tree and locally treelike graph without boundary, Phys. Rev. B 94, 184203 (2016).

[34] K. S. Tikhonov and A. D. Mirlin, Statistics of eigenstates near the localization transition on random regular graphs, Phys. Rev. B 99, 024202 (2019).

[35] S. Bera, G. De Tomasi, I. M. Khaymovich, and A. Scardicchio, Return probability for the Anderson model on the random regular graph, Phys. Rev. B 98, 134205 (2018).

[36] V. E. Kravtsov, I. M. Khaymovich, B. L. Altshuler, and L. B. Ioffe, Localization transition on the random regular graph as an unstable tricritical point in a log-normal RosenzweigPorter random matrix ensemble, arXiv:2002.03979.

[37] Y. Jia and J. J. M. Verbaarschot, Chaos on the hypercube, J. High Energy Phys. 11 (2020) 154.

[38] J. Maldacena and X. L. Qi, Eternal traversable wormhole, arXiv:1804.00491.

[39] A. M. García-García, T. Nosaka, D. Rosa, and J. J. M. Verbaarschot, Quantum chaos transition in a two-site Sachdev-Ye-Kitaev model dual to an eternal traversable wormhole, Phys. Rev. D 100, 026002 (2019).

[40] J. Maldacena, Eternal black holes in anti-de Sitter, J. High Energy Phys. 04 (2003) 021.

[41] M. Van Raamsdonk, Comments on quantum gravity and entanglement, Gen. Relativ. Gravit. 42, 2323 (2010).

[42] A. May and M. Van Raamsdonk, Interpolating between multi-boundary wormholes and single-boundary geometries in holography, arXiv:2011.14258.

[43] Ab. Al. Balushi, Z. Wang, and D. Marolf, Traversability of multi-boundary wormholes, arXiv:2012.04635.

[44] J. Maldacena and L. Maoz, Wormholes in AdS, J. High Energy Phys. 02 (2004) 053.

[45] A. Jahn, M. Gluza, F. Pastawski, and J. Eisert, Majorana dimers and holographic quantum error-correcting codes, Phys. Rev. Research 1, 033079 (2019).

[46] M. Hanada and J. Maltz, A proposal of the gauge theory description of the small Schwarzschild black hole in ADS5xS5, J. High Energy Phys. 12 (2017) 012.

[47] J. S. Cotler, G. Gur-Ari, M. Hanada, J. Polchinski, P. Saad, S. H. Shenker, D. Stanford, A. Streicher, and Masaki Tezuka, Black holes and random matrices, J. High Energy Phys. 05 (2017) 118.

[48] F. Viger and M. Latapy, Efficient and simple generation of random simple connected graphs with prescribed degree sequence, computing and combinatorics, Lect. Notes Comput. Sci. 3595, 440 (2005).

[49] D. Marolf and H. Maxfield, Transcending the ensemble: Baby universes, spacetime wormholes, and the order and disorder of black hole information, J. High Energy Phys. 08 (2020) 044. 
[50] P. Saad, S. H. Shenker, and D. Stanford, JT gravity as a matrix integral, arXiv:1903.11115.

[51] T. Banks, W. Fischler, S. H. Shenker, and L. Susskind, M theory as a matrix model: A conjecture, Phys. Rev. D 55, 5112 (1997).

[52] O. Aharony, J. Marsano, S. Minwalla, K. Papadodimas, and M. Van Raamsdonk, The Hagedorn-deconï $\neg$ nement phase transition in weakly coupled large $\mathrm{N}$ gauge theories, Adv. Theor. Math. Phys. 8, 603 (2004).

[53] D. Berenstein, Submatrix deconï $\neg$ nement and small black holes in AdS, J. High Energy Phys. 09 (2018) 054.

[54] M. Hanada, A. Jevicki, C. Peng et al., Anatomy of deconfinement, J. High Energy Phys. 02 (2019) 167.

[55] M. Hanada, G. Ishiki, and H. Watanabe, Partial deconfinement, J. High Energy Phys. 03 (2019) 145.

[56] G. De Tomasi, I. M. Khaymovich, Fr. Pollmann, and S. Warzel, Rare thermal bubbles at the many-body localization transition from the Fock space point of view, arXiv:2011 .03048 .

[57] S. Moudgalya, St. Rachel, B. A. Bernevig, and N. Regnault, Exact excited states of nonintegrable models, Phys. Rev. B 98, 235155 (2018).

[58] C. J. Turner, A. A. Michailidis, D. Abanin, M. Serbyn, and Zl. Papic, Weak ergodicity breaking from quantum manybody scars, Nat. Phys. 14, 745 (2018).

[59] P. Sala, T. Rakovszky, R.Verresen, M. Knap, and Fr. Pollmann, Ergodicity-Breaking Arising from Hilbert Space
Fragmentation in Dipole-Conserving Hamiltonians, Phys. Rev. X 10, 011047 (2020).

[60] V. Khemani and R. Nandkishore, Local constraints can globally shatter Hilbert space: A new route to quantum information protection, Phys. Rev. B 101, 174204 (2020).

[61] N. Shiraishi and T. Mori, Systematic Construction of Counterexamples to the Eigenstate Thermalization Hypothesis, Phys. Rev. Lett. 119, 030601 (2017).

[62] K. Pakrouski, P. N. Pallegar, F. K. Popov, and I. R. Klebanov, Many Body Scars as a Group Invariant Sector of Hilbert Space, Phys. Rev. Lett. 125, 230602 (2020).

[63] L. Herviou, J. H. Bardarson, and N. Regnault, Many-body localization in a fragmented Hilbert space, arXiv:2011.04659.

[64] D. Basko, Weak chaos in the disordered nonlinear Schroedinger chain: Destruction of Anderson localization by Arnold diffusion, Ann. Phys. (Amsterdam) 326, 1577 (2011).

[65] This result is obtained in collaboration with A. Vasyliev.

[66] J. B. Geloun and S. Ramgoolam, Quantum mechanics of bipartite ribbon graphs: Integrality, Lattices and Kronecker coefficients, arXiv:2010.04054.

[67] B. Swingle, Constructing holographic spacetimes using entanglement renormalization, arXiv:1209.3304.

[68] Sh. Xu, L. Susskind, Y. Su, and B. Swingle, A sparse model of quantum holography, arXiv:2008.02303.

[69] J. J. M. Verbaarschot and T. Wettig, Random matrix theory and chiral symmetry in QCD, Annu. Rev. Nucl. Part. Sci. 50, 343 (2000). 